\title{
Ocular tissue for transplantation- fresh, chilled, warmed, frozen or pickled?
}

Eye (2004) 18, 865-866. doi:10.1038/sj.eye.6701344

It might appear disrespectful to compare ocular tissue storage with the food industry, but to do so allows the recognition of some features they have in common. While acknowledging the scientific contribution to the improvement of techniques in both areas, the range of methods used for preservation and storage have largely developed empirically, evolving over time. This can make it difficult to implement change as there is always likely to be an understandable reluctance to alter methods 'that work'.

Sclera for transplantation has a long shelf life in alcohol. Limbal tissue is currently thought to be at its best when 'fresh' (ie, whole eyes stored for less than $48 \mathrm{~h}$ ) or after in vitro expansion. Cryopreservation is not yet sufficiently developed to be of practical use ${ }^{1}$ though its potential could be substantial for tissueengineered grafts. Full- or partial-thickness corneal transplants are the most common procedures undertaken where donor tissue is stored either at $4^{\circ} \mathrm{C}$ or in organ culture at approximately physiological temperature. The clinical results from hypothermic storage and organ culture are comparable but while the former is technically simple, organ culture offers a considerably extended storage time. Both techniques are used in the UK and a symbiotic relationship has existed for many years, whereby large numbers of corneas in hypothermic storage media have been sent from Moorfields Eye Hospital, Norwich, East Grinstead and, more recently, Liverpool and Newcastle to the eye banks at Bristol and Manchester for extended storage by organ culture. Thus, in the UK, this network of donor centres, coordinated through UK Transplant, allows $>90 \%$ of ocular tissue requirements for a population of 60 million to be met by just two

AB Tullo and WJ Armitage

eye banks using organ culture

(www.uktransplant.org.uk). Only recently have there been any consistent attempts to rationalize these methods, in use since the 1970s.

Hypothermic storage was advanced

considerably with the introduction of Optisol in the 1990s, and improvements to the composition of organ culture medium are now being actively sought. ${ }^{2}$

Since virtually all eyes coming to an eye bank will have bacteria and fungi contaminating the ocular surface, ${ }^{3}$ there has been much debate of the relative merits of organ culture $v s$ hypothermia. Cleaning protocols prior to storage substantially reduce this microbial load, and the antibiotics in organ culture medium will be in the temperature range where they are most effective. There is also a greater opportunity of detecting persistent microbes, reducing the risk that contaminated corneas will be transplanted. Retrospective comparisons suggest that this translates into a lower risk of postoperative endophthalmitis, although confirmation of this through a definitive, prospective study is awaited.

An advantage of hypothermic storage, where the aim is to suppress the metabolic and energy requirements of the tissue, is that it uses defined media. On the other hand, organ culture aims to support cellular metabolism and currently relies on medium supplemented by bovine serum. Attempts to replace the serum, which contains a range of active components, are underway, but this is not a trivial problem; in the meantime, serum is sourced from countries where no BSE has been reported. Other improvements could come from preventing stromal oedema and minimizing changes in composition of the medium such as falling $\mathrm{pH}$ and glucose levels, all of which are stressful to the cornea. Given the microbiological circumstances of organ culture,
Manchester Royal eye Hospital Oxford Road, M13 9WH UK

Correspondence: AB Tullo Tel: + 4416125624223

Fax: + 441612726618

E-mail: andrew.tullo@ cmmc.nhs.uk

Received: 18 September 2003

Accepted: 18 September 2003 
it has also been suggested that endotoxins may have a deleterious effect on corneas. In this issue of Eye, Spelsberg et $a l^{4}$ in Dusseldorf examined this potential influence of endotoxins. They are to be congratulated not only on submitting data that suggests the endotoxins are not influential but arrived at this conclusion by studying clinical outcome.

There has been substantial improvement in Europe in the organizational aspects of procurement and storage of ocular tissue (www.eeba.net). Recently, the routine accrual of clinical outcome data in the UK is being undertaken with impressive return rates, thanks to the joint efforts of UK Transplant, the Royal College of Ophthalmologists and participating surgeons. Such data are crucial for the implementation and validation of improved methodology and techniques. We cannot yet bank on catering for all the needs of surgeons who wish to transplant ocular tissue effectively, but there are continuing opportunities to do so.

\section{References}

1 Armitage WJ, Hall SC, Routledge C. Recovery of endothelial function after vitrification of cornea at $-110^{\circ} \mathrm{C}$. Invest Ophthalmol Vis Sci 2002; 43: 2160-2164.

2 Thuret G, Chiquet C, Bernal F, Acquart S, Romanet J-P, Mouillon $\mathrm{M}$ et al. Prospective, randomised clinical and endothelial evaluation of 2 storage times for corneal donor tissue in organ culture at $31^{\circ} \mathrm{C}$. Arch Opthalmol 2003; 121: $442-450$.

3 Albon J, Armstrong M, Tullo AB. Bacterial contamination of human organ cultured corneas. Cornea 2001; 20: 260-263.

4 Spelsberg H, Reinhard T, Sokolovska Y, Zysk G, Bohrinnger $D$, Sundmacher R. Endotoxin is storage medium of human corneal grafts and clinical course after penetrating normalrisk keratoplasty. Eye 2004; (in press). 